\title{
Correction to: IGHV mutational status of nodal marginal zone lymphoma by NGS reveals distinct pathogenic pathways with different prognostic implications
}

\author{
Massimo Granai ${ }^{1} \cdot$ Teresa Amato $^{1} \cdot$ Arianna Di Napoli $^{2} \cdot$ Raffaella Santi $^{3} \cdot$ Federica Vergoni $^{3} \cdot$ Gioia Di Stefano $^{3}$. \\ Virginia Mancini ${ }^{1}$. Sofya Kovalchuk ${ }^{4}$. Emanuele Cencini ${ }^{5}$ - Alberto Giulio Carta ${ }^{1}$ - Sara Aversa ${ }^{1}$. Marita Ziepert ${ }^{6}$. \\ Gabriele Cevenini ${ }^{1}$ - Stefano Lazzi ${ }^{1}$ • Lorenzo Leoncini ${ }^{1}$ • Cristiana Bellan ${ }^{1}$
}

Published online: 18 March 2020

(C) Springer-Verlag GmbH Germany, part of Springer Nature 2020

\section{Correction to: Virchows Archiv}

https://doi.org/10.1007/s00428-019-02712-8

This error was caused due to the author's oversight and this does not change the views or the results presented in the manuscript.

The original article has been corrected.

Publisher's note Springer Nature remains neutral with regard to jurisdictional claims in published maps and institutional affiliations.

The online version of the original article can be found at https://doi.org/ 10.1007/s00428-019-02712-8

Lorenzo Leoncini

lorenzo.leoncini@dbm.unisi.it

1 Department of Medical Biotechnologies, Anatomic Pathology Division, University of Siena, Via delle Scotte, 6, 53100 Siena, Italy

2 Department of Clinical and Molecular Medicine, Pathology Unit, University of Rome "La Sapienza", Rome, Italy

3 Florence Pathology Unit, Careggi University Hospital, Florence, Italy

4 Florence Hematology Unit, University of Florence, Florence, Italy

5 Department of Hematology, University of Siena, Siena, Italy

6 Institute for Medical Informatics, Statistics and Epidemiology, University of Leipzig, Leipzig, Germany 